\begin{tabular}{|l|c|c|c|}
\hline Eiszeitalter u. Gegenwart & $\mathbf{2 8}$ & $\begin{array}{c}139-147 \\
4 \mathrm{Abb} .\end{array}$ & Öbringen/Württ. 1978 \\
\hline
\end{tabular}

\title{
Neue Beobachtungen zur Chronostratigraphie der mittelwisconsinzeitlichen Vergletscherungen und Böden mexikanischer Vulkane *)
}

\author{
KLAus Heine **)
}

Upper Pleistocene, glaciations, fossil soils, chronostratigraphy, Central Mexican Highland

Kurzfassung: Die chronostratigraphische Stellung einer mittelwisconsinzeitlichen Moräne des Nevado de Toluca/Mexiko wird beschrieben und mit früheren Stratigraphien verglichen. Der $26000-21000$ a B.P.-Boden des zentralmexikanischen Hochlandes kann durch absolute $\mathrm{Da}$ tierungen nicht nur am Malinche-Vulkan, sondern auch in der Sierra Nevada (Río Frio) und am Nevado de Toluca belegt werden. Beide Beobachtungen bestätigen die früheren stratigraphischen Beobachtungen des Verfassers.

[New observations about the chronostratigraphy of the mid-Wisconsinian glaciations and soils of the Mexican volcanoes]

A bstract: The chronostratigraphical position of a mid-Wisconsin moraine of the Nevado de Toluca volcano/Mexico is described and compared with former stratigraphies. The 26,000$21,000 \mathrm{yr}$ B.P. soil of the central Mexican highland cannot only be proved at La Malinche volcano but also in the Sierra Nevada de México (Río Frio) and at the Nevado de Toluca volcano by radiocarbon determinations. Both observations confirm the former stratigraphical works of the author.

\section{Einleitung}

Verf. hat während der letzten Jahre wiederholt über stratigraphische Probleme der jungquartären Sedimente aus dem Bereich der zentralmexikanischen hohen Vulkane berichtet (Heine 1971, 1973a + b, 1974a, 1975, 1976a; Heine \& Heide-Weise 1972, 1973 ; Heine \& Schöhals 1973; Heine \& Ohngemach 1976). Während die stratigraphischen Beziehungen der glazialen und periglazialen Ablagerungen und Formen für die Zeit nach 25000 a B.P. recht gut erfaßt werden konnten, gab es bisher wenig Anhaltspunkte für die stratigraphische Stellung mittelwisconsinzeitlicher Moränen, die bisher nur am MalincheVulkan anhand sedimentologischer und paläopedologischer Kriterien in Verbindung mit wenigen ${ }^{14} \mathrm{C}$-Datierungen aus liegenden und hangenden Schichten der Moränen (M I) auf rund 34000 bis 32000 a B.P. datiert werden konnten. Neue Beobachtungen des Verfassers aus den Jahren 1974 und 1975 ergänzen und bestätigen die früheren Befunde.

Für jungquartäre stratigraphische Korrelierungen im Bereich des südlichen zentralmexikanischen Hochlandes ist ein fossiler Boden, der von mir mit $\mathrm{fBo} 1$ bezeichnet wurde (HeINE 1971, 1975), von großer Bedeutung. Darauf wurde von mir an verschiedenen Stellen hingewiesen. Während bisher absolute Datierungen dieses fBo1-Bodens nur aus dem Bereich des Malinche-Vulkans vorlagen, können nun die aufgrund der Feldbeobachtungen vorgenommenen Korrelierungen des fBo1-Bodens über größere Entfernungen auch durch verschiedene ${ }^{14} \mathrm{C}$-Datierungen bestätigt werden. Das Alter von rund 26000 bis 21000 a B.P. für den fBo1-Boden wird auch durch die neuen Befunde gestützt.

*) Der Deutschen Forschungsgemeinschaft danke ich für eine großzügige Reise- und Sachbeihilfe. Herrn Professor Dr. M.-A. G e y h vom Niedersächsischen Landesamt für Bodenforschung (Hannover) danke ich für zahlreiche $14 \mathrm{C}$-Datierungen.

**) Anschrift des Verfassers: Prof. Dr. Klaus $\mathrm{H}$ e in e, Geographisches Institut der Universität, Franziskanerstraße 2, D-5300 Bonn 1. 


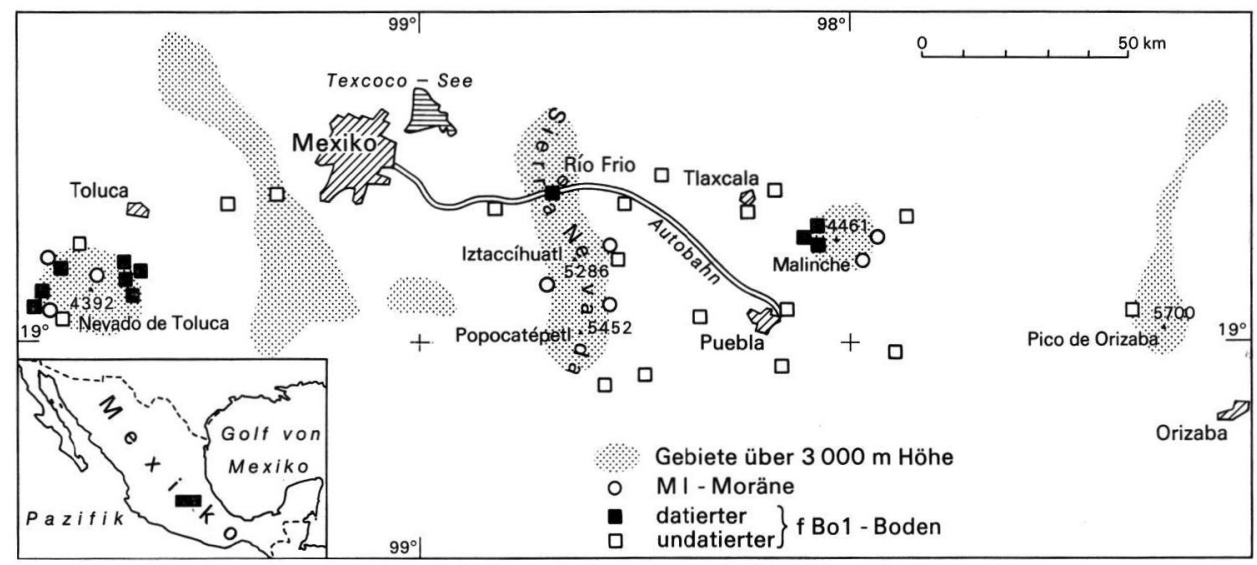

Abb. 1: Übersichtskärtchen.

\section{Geländebeobachtungen}

\subsection{Die M I-Moränen des Nevadode Toluca}

Über die chronostratigraphischen Beziehungen der glazialen und periglazialen Formen und Sedimente Zentralmexikos informiert die Zusammenstellung der Abb. 2. Am Vulkan des Nevado de Toluca (Abb. 1) konnten bisher die M I-, M III-, M IV- und M VVergletscherungen, die durch Moränen und Blockgletscher vertreten sind, nachgewiesen werden (HEINE 1975, 1976b); anhand morphologischer Kriterien und relativ-stratigraphischer Beziehungen wurden die glazialen Formen und Ablagerungen der M I- und M III-Vergletscherungen mit den entsprechenden datierten Bildungen anderer Vulkane korreliert (HEINE 1975).

Am Nordwesthang des Nevado de Toluca waren in ca. 3820 m Höhe am 22. 2. 1975 Aufschlüsse entlang der Fahrstraße zum Kraterkessel vorhanden, die Einblicke in die Schichtabfolge gewährten (Abb. 3). Im Liegenden befanden sich einerseits glaziale Ablagerungen (Moränen in ca. $3900 \mathrm{~m}$ Höhe, rechtes Profil der Abb. 3), andererseits in derselben stratigraphischen Position vulkanische andesitische Brekzien (Lahar, mittleres Profil der Abb.3). Sowohl die Moräne als auch die Laharablagerungen wurden von derselben Abfolge verschiedener, vorwiegend vulkanischer Sedimente bedeckt. Aus dem Laharmaterial, dessen größere Komponenten eine leichte Einregelung hangabwärts zeigten, konnten verschiedene Holzkohlestücke geborgen werden, die zum Teil über $20 \mathrm{~cm}$ Länge aufwiesen und die auch teilweise eine Einregelung hangabwärts erkennen ließen. Das Alter der ${ }^{14} \mathrm{C}$ Datierung ergab $35600 \pm 2600$ a B.P. (Hv 6959).

Aufgrund der geringen Bodenbildung auf dem Laharmaterial sowie dem Moränenmaterial und derselben Tephrabedeckung von Lahar und Moräne wird angenommen, daß die Moräne nur etwas jünger als das Laharmaterial sein kann; dafür spricht auch die Tatsache, daß das Moränenmaterial nicht vom Lahar bedeckt worden ist. Die Moräne ist damit ein Beleg für eine mittelwisconsinzeitliche Vergletscherung des Nevado de Toluca.

Vermutlich ebenfalls mittelwisconsinzeitliche glaziale Ablagerungen wurden an zwei anderen Stellen des Nevado de Toluca gefunden (Heine 1975); sie sind in Höhen von 3200 und $3000 \mathrm{~m}$ angetroffen worden und zeigen eine Bedeckung mit verschiedenen vulkanischen Schichten; der fossile fBo1-Boden tritt auch bei den zuletzt genannten Moränen unmittelbar über dem Moränenschutt auf. 


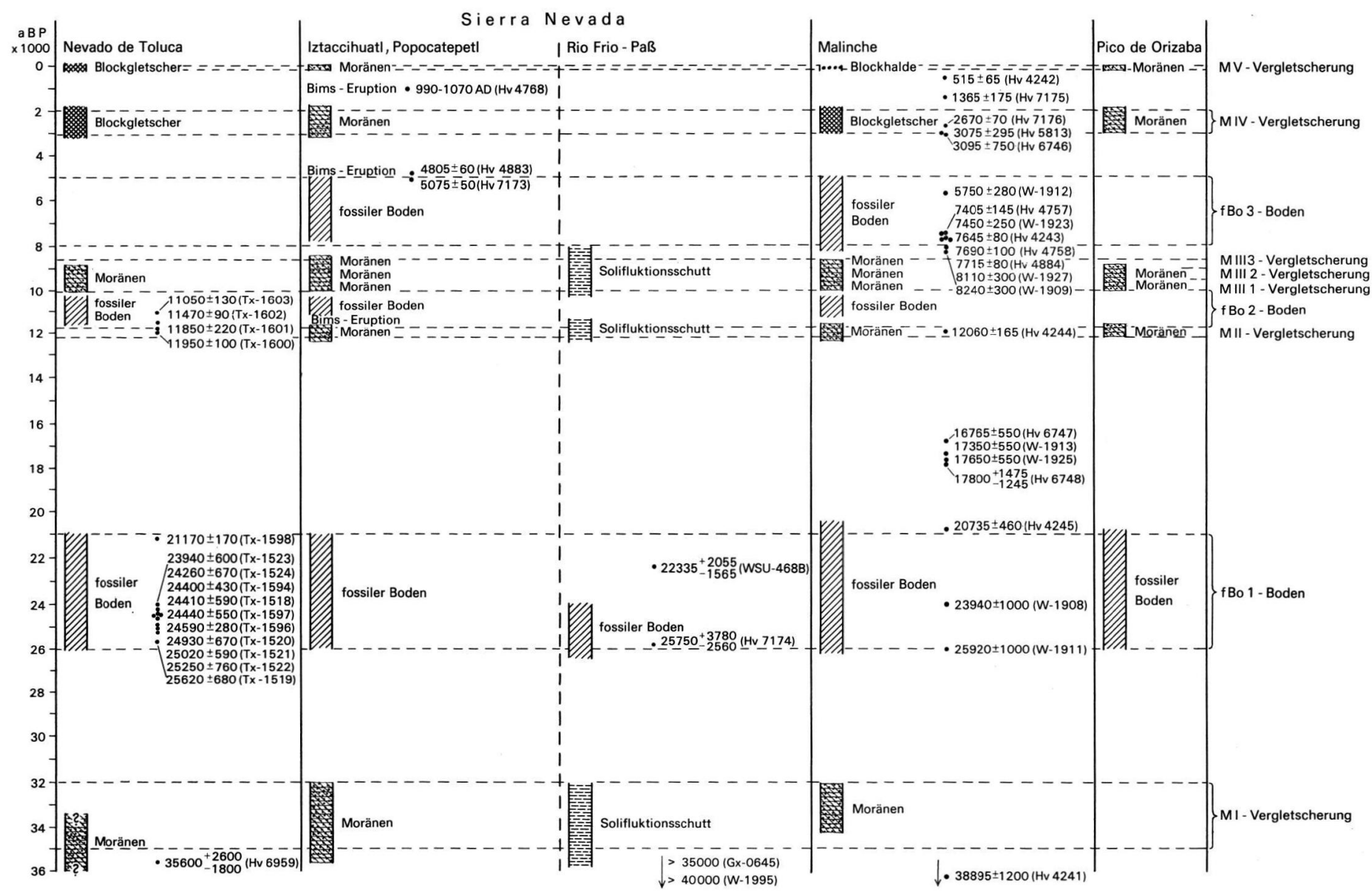

Abb. 2: Chronostratigraphische Beziehungen der glazialen und periglazialen Formen und Ablagerungen in Zentralmexiko (schematisch). Die ${ }^{14} \mathrm{C}$-Datierungen mit den Tx-Probennummern des Nevado de Toluca sind der Arbeit von Bloomfield \& VAlastroo (1974) entnommen. Die Darstellung enthält alle für die genannten Vulkangebirge verfügbaren ${ }^{14} \mathrm{C}$-Datierungen. 


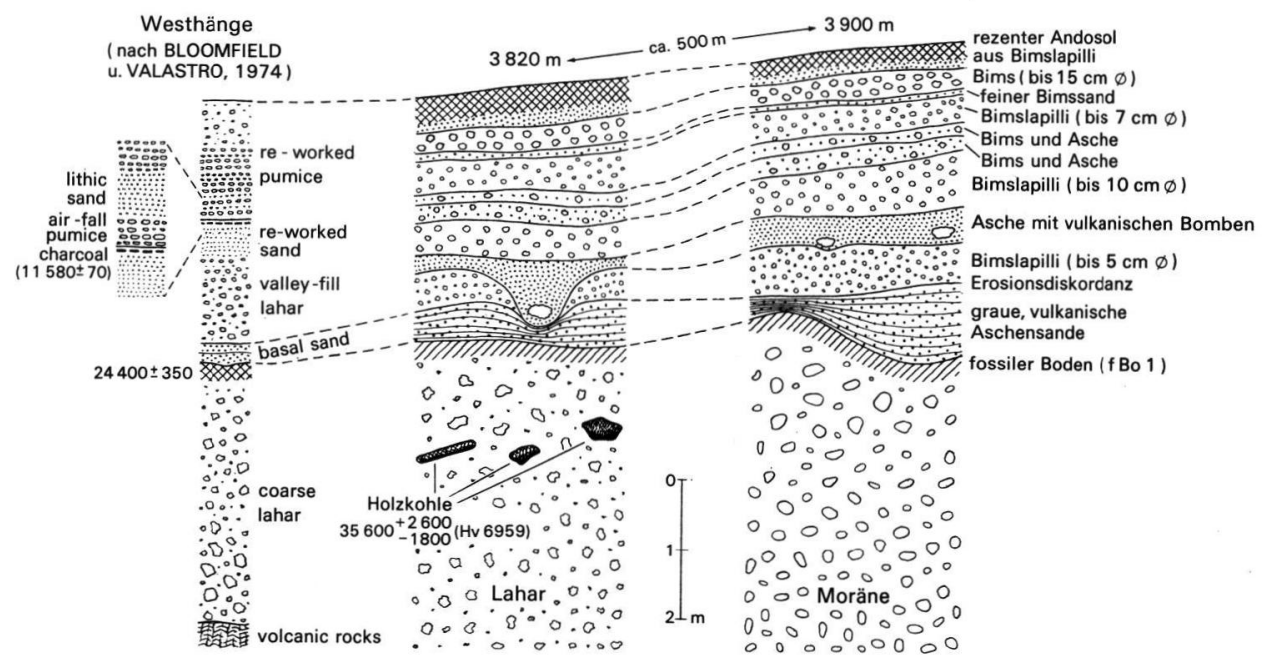

Abb. 3: Profil und stratigraphische Beziehungen der M I-Moräne des NW-Hanges des Nevado de Toluca. Die eigenen Profile lassen sich mit dem Sammelprofil für die Westflanke des Vulkans, wie es von Boomflied \& Valastro (1974) angegeben wird, korrelieren.

\subsection{Der fBo1-Boden am Nevadode Toluca}

Bloomfield \& Valastro (1974) machen Angaben über die spätpleistozäne Förderfolge des Nevado de Toluca, die sie mit zahlreichen ${ }^{14} \mathrm{C}$-Datierungen belegen (Abb.2). Ihre erarbeiteten Tephra-Sequenzen lassen sich mit meinen früheren und den hier dargestellten Profilen korrelieren (Abb. 3). Der von mir als fBo1-Boden (= 25000 a B.P.-Boden) bezeichnete und für stratigraphische Fern-Korrelierungen zwischen Malinche und Sierra $\mathrm{Ne}-$ vada einerseits und dem Nevado de Toluca andererseits benutzte fossile Boden wurde von den genannten Autoren durch $11{ }^{14} \mathrm{C}$-Datierungen chronostratigraphisch eingeordnet. Die Altersbestimmungen ergaben Werte zwischen rund 26000 und 21000 a B.P. und entsprechen damit genau dem Alter des fBo1-Bodens des Malinche-Vulkans (Heine 1973b: Abb. 2).

Durch die absoluten Datierungen des fBo1-Bodens am Nevado de Toluca wird auch gleichzeitig das Alter der M I-Moräne auf älter als 26000 a B.P. festgelegt. Ob allerdings die Ablagerungen des älteren Laharstromes ein Alter von nur rund 25000 a B.P. haben, wie Bloomfield \& Valastro (1974) aufgrund der fBo1-Boden-Datierungen vermuten, muß angezweifelt werden, denn die Datierung des Laharmaterials in unmittelbarer Nachbarschaft zum Moränenschutt auf $35600 \pm 2600$ a B.P. läßt zumindest für den datierten Lahar ein wesentlich höheres Alter erkennen.

\subsection{Der fBo1-Boden im Bereich des Río Frio-Passes}

(Sierra Nevada de México)

Aufgrund paläopedologischer und mineralogischer Befunde ist wiederholt versucht worden, stratigraphische Beziehungen zwischen den Sedimenten des Beckens von Mexiko und des Tales von Puebla-Tlaxcala herzustellen. Dabei kam den Aufschlüssen entlang der Autobahn, die beide Beckengebiete verbindet (Abb. 1), im Bereich der Sierra Nevada eine 
besondere Bedeutung zu (Cornwall 1968, 1970; Heine \& Schönhals 1973; Heine 1975). Nicht ganz übersichtlich sind die Verhältnisse im Bereich des Río Frio-Passes nördlich des Iztaccíhuatl-Vulkangebirges, denn dort schneidet die Autobahn verschiedene jüngere Lavaströme, Ignimbrite, Schutthorizonte, fossile Böden und Tephralagen, die in Abhängigkeit von dem bewegten Relief unterschiedlich mächtig oder auch nur lückenhaft von Ort zu Ort ausgebildet sind. Eine erneute Untersuchung der Río Frio-Profile unter besonderer Berücksichtigung der periglazialen Sedimente ergab neue Kriterien für die chronostratigraphische Stellung des fBo1-Bodens im Bereich des Río Frio-Gebietes und der Westseite der Sierra Nevada sowie für die Altersstellung der Río Frio-Ignimbrite.

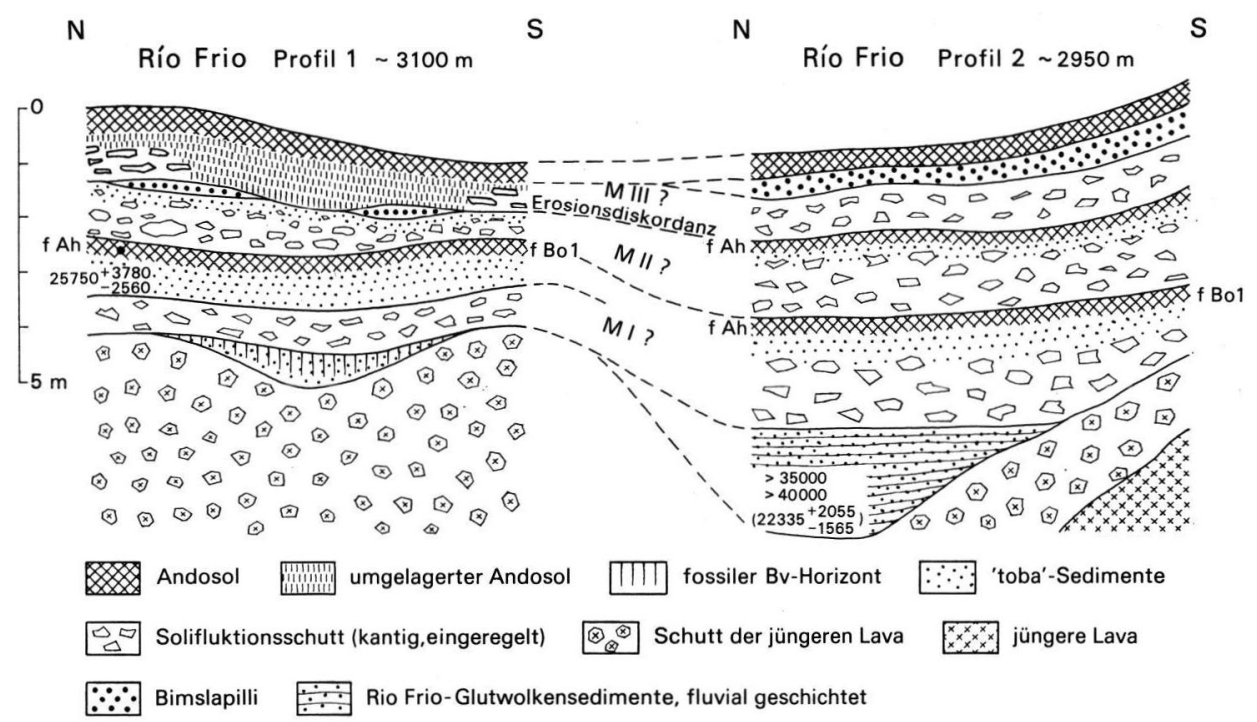

Abb. 4: Profile und stratigraphische Beziehungen der Solifluktionshorizonte sowie der Glutwolkensedimente im Bereich des Río Frio-Passes, Sierra Nevada de México.

Zwei Profile sollen herausgegriffen und näher beschrieben werden (Abb. 4). Profil 1 befindet sich in rund $3100 \mathrm{~m}$ Höhe westlich der Paßhöhe; Profil 2 liegt zwischen der Paßhöhe und dem Ort Río Frio in ca. 2950 m Höhe. Im Liegenden von Profil 1 erkennt man den Schutt der Jüngeren Lava (nach CoRnwall 1968); der Lavastrom selbst liegt etwas weiter südlich des Profils. Über dem Lavaschutt ist in Mulden Bodenmaterial eingeschwemmt worden. Im Hangenden folgt ein Solifluktionshorizont mit eingeregelten Komponenten; der Horizont ist 0,5 bis $2 \mathrm{~m}$ mächtig und enthält kantige Scherben und Blöcke, die bis $1 \mathrm{~m} \phi$ erreichen können. Darüber folgen im Profil braun verwitterte "toba"-Sedimente, auf denen ein Andosol mit ausgeprägtem $\mathrm{A}_{\mathrm{h}}$-Horizont entstanden ist. Kleine Holzkohlestückchen befinden sich in dem fossilen Andosol. Holzkohle- und Humusbestandteile bildeten das Material für eine ${ }^{14} \mathrm{C}$-Datierung, die ein Alter von $25750 \pm \begin{aligned} & 3780 \\ & 2560\end{aligned}$ a. B.P. (Hv 7174) ergab. Über dem datierten fossilen Andosol liegt ein zweiter Solifluktionshorizont, der ebenfalls aus kantigen Bruchstücken des nahegelegenen Lavastromes (Jüngere Lava) besteht; die größten Komponenten in dem 0 bis 1,5 m mächtigen Solifluktionsschutt haben einen $\phi$ bis zu $1 \mathrm{~m}$. Im Hangenden nimmt das "toba“-Material im Solifluktionshorizont mehr und mehr zu; die „toba“-Sedimente sind leicht verwittert. Eine Erosionsdiskordanz trennt die nur in Linsen auftretenden Bimslapilli von dem liegenden (mittleren) Solifluk- 
tionsschutt. Über den Bimslinsen ist stellenweise Solifluktionsschutt abgelagert, stellenweise aber auch humushaltiges schluffig-sandiges Material, das als umgelagerter AndoBoden gedeutet werden muß. Darüber befindet sich - bis zu $1 \mathrm{~m}$ mächtig - der rezente Andosol.

In Profil 2 sind die Beziehungen zwischen dem Schutt der Jüngeren Lava und dem Río Frio-Ignimbrit einerseits und dem Ignimbrit und den Solifluktionshorizonten andererseits zu erkennen. Der Ignimbrit wird durch fluvial umgelagerte, geschichtete Sedimente in diesem Profil vertreten. Etwa $500 \mathrm{~m}$ nördlich des Profils 2 steht der Ignimbrit südlich des Dorfes Río Frio an, wo er auch verschiedene verkohlte Kiefernstämme enthält, die unterschiedliche ${ }^{14}$ C-Alter ergeben haben (CoRnwall 1968, 1970; Hrine 1973b). Der Bimslinsenhorizont zwischen den beiden hangenden Solifluktionshorizonten des Profils 1 ist in Profil 2 nicht mehr aufzufinden. Hier tritt jedoch ein wesentlich gröberer Bims ( $\phi$ bis $2 \mathrm{~cm}$ ) in einer 0 bis $1 \mathrm{~m}$ mächtigen Schicht über dem jüngsten Solifluktionsschutt auf.

Beide Profile zeigen drei deutlich voneinander abgesetzte Solifluktionshorizonte, die auch noch in Höhen unter $3000 \mathrm{~m}$ ausgebildet sind. Die beiden untersten Solifluktionslagen werden von einem fossilen Boden getrennt, der ein ${ }^{14} \mathrm{C}$-Alter von rund 25000 a B.P. hat und damit dem fBo1-Boden des Malinche-Vulkans (HeIne 1975) und des Nevado de Toluca entspricht. Aufgrund der stratigraphischen Beziehungen der Solifluktionshorizonte zu dem fBo1-Boden lassen sich diese mit der Moränen-Stratigraphie korrelieren (Abb. 2), die für die zentralmexikanischen Vulkane erarbeitet wurde. Demnach entspricht vermutlich der unterste Solifluktionsschutt der M I-Vergletscherung, der mittlere Solifluktionsschutt der M II-Vergletscherung und der oberste Schutt der M III-Vergletscherung.

Der Río Frio-Ignimbrit ist im Liegenden des M I-Solifluktionshorizontes zu finden; er hat somit ein Alter von über rund 35000 a B.P.

\section{Diskussion der Ergebnisse}

Hinweise auf eine mittelwisconsinzeitliche Vergletscherung verschiedener mexikanischer Vulkane (Malinche, Sierra Nevada und Nevado de Toluca) hat Verf. bereits in früheren Publikationen gegeben (zuletzt HeINe 1975). Bisher konnte jedoch die Datierung dieser sogenannten M I-Vergletscherung nur aufgrund sedimentologischer und paläopedologischer Kriterien vorgenommen werden. Das vermutete Alter für die M I-Vergletscherung von ca. $34000-32000$ a B.P. wird durch die hier erstmals erwähnte ${ }^{14} \mathrm{C}$-Datierung im großen und ganzen bestätigt. Wahrscheinlich ist mit einem Alter von ca. 35000 a B.P. oder etwas jünger für die M I-Moräne des Nevado de Toluca zu rechnen.

Damit fällt die M I-Vergletscherung des Nevado de Toluca (und vermutlich auch die der anderen mexikanischen Vulkane) in eine Zeit, die ein mittelwisconsinzeitliches Stadial, bzw. den Übergang von diesem Stadial zum bedeutenden Interstadial (Denekamp, Stillfried B, Karginsky, Plum Point etc. nach Mörner 1971, 1972, 1973) verkörpert. Auch für tropische und subtropische Gebiete scheint um 35000 bis 32000 a B.P. eine bedeutende Klimaschwankung thermischer und/oder hygrischer Art nachgewiesen zu sein (HeINE 1974b; van Zinderen baKker \& COetzee 1972 für das tropische Afrika; Bowler 1975 für Australien). Aufgrund der Ungenauigkeiten mittelwisconsinzeitlicher ${ }^{14} \mathrm{C}-\mathrm{Datierun}$ gen läßt sich nicht sagen, ob die M I-Vergletscherung mit einem außertropischen Stadial i.e.S. korrespondiert oder ob sie möglicherweise erst in der Zeit des Überganges vom Stadial zum Interstadial auftrat - analog den Verhältnissen im Spätglazial/Frühholozän, wie sie für die Vergletscherungen M II und M III der mexikanischen Gebirge belegt werden konnten (Heine 1975; HeINe \& OHNGemach 1976). In der Zeit nach 25000 a B.P. waren in Mexiko die größten Gletschervorstöße nicht zeitgleich mit den Hauptvergletsche- 
rungen der Außertropen, sondern traten erst am Übergang vom Pleistozän auf (M II um 12000 a B.P., M III um 10000 - 9000 a B.P.). Erklärungen für dieses Phänomen geben Klaus (1973) und Heine (1977).

Ein fossiler Boden, der fBo1-Boden (nach Heine 1971), der ein Alter von 26000 bis 21000 a B.P. hat, besitzt für stratigraphische Korrelierungen im zentralmexikanischen Hochland eine große Bedeutung. Dieser fBo1- Boden konnte bisher nur am Malinche-Vulkan datiert werden (HeINe 1975); nun liegen auch Datierungen vom Nevado de Toluca und der Sierra Nevada (Río Frio) vor. Die Befunde bestätigen die früheren Folgerungen, die allein anhand der Geländebefunde gemacht wurden (Heine 1975). Der fBo1-Boden verkörpert eine Zeit, die durch Stabilität der morphologischen Prozesse gekennzeichnet ist. Der fBo1-Boden ist sowohl an den Hängen der Vulkane als auch in den Beckenlandschaften ausgebildet. Er belegt Abtragungsruhe zwischen 26000 und 21000 a B.P. Da dieser Boden erst in der ausgehenden Wisconsin-Kaltzeit von Sedimenten bedeckt bzw. abgetragen wurde (HEINE 1976a), verkörpert er auch über weite Flächen im zentralmexikanischen Hochland das letzteiszeitliche Relief zwischen ca. 21000 und 13000 a B.P. Diese Zeitspanne ist in Mexiko durch ein kaltes und trockenes Klima charakterisiert (KLAUS 1973; HEINE 1975).

Die Identifizierung des fBo1-Bodens in den Río Frio-Profilen ergibt neue Anhaltspunkte für die stratigraphische Stellung des Río Frio-Ignimbrits sowie der „toba“-Sedimente an der Westflanke der Sierra Nevada. Die Altersangaben der Ignimbritablagerungen sind recht unterschiedlich (HeIne 1973b); MALDE (o. J.) nennt $22335 \pm \begin{aligned} & 2055 \\ & 1565\end{aligned}$ a. B.P. (WSU-468B), über 35000 (GX-0645) und über 40000 (W-1995) a B.P. Mit Sicherheit kann nun das Alter der Ignimbrite auf über 35000 a B.P. festgesetzt werden, da der fBo1Boden sowie die mit verschiedenen datierten Vergletscherungsphasen korrelierbaren Solifluktionshorizonte die Glutwolkensedimente bedecken. Daher müssen stratigraphische Beziehungen des fBo1-Bodens bei Heine (1973b) und Heine \& Schönhals (1973) berichtigt werden, die für das Gebiet des Río Frio-Passes und der westlichen Sierra Nevada auf Ergebnisse von CoRNWall $(1968,1970)$ zurückgreifen; CoRNwall hat die Beziehungen der fossilen Böden zwischen dem Río Frio-Paß und den Beckengebieten nicht richtig erkannt, wie die hier ausgeführten speziellen Untersuchungen ergeben haben. Der fBo1-Boden ist am Río Frio-Paß nicht mit dem ,First Red Soil' CoRnwalls (1968) identisch, wohl aber mit dem ,First Red Soil' im Bereich des Beckens von Puebla-Tlaxcala.

Der fossile Boden fBo1 gliedert im Bereich des Río Frio-Passes einen unteren Solifluktionshorizont von zwei hangenden Solifluktionsdecken. Die stratigraphische Lage der Solifluktionshorizonte legt die Vermutung nahe, daß es sich bei den Solifluktionsdecken um korrelate Ablagerungen zu den Moränen M I, M II und M III handelt, die an den zentralmexikanischen Vulkanen nachgewiesen und chronostratigraphisch erfaßt wurden. Die M I-Moränen befinden sich stets unter dem fBo1-Boden; sie haben ein Alter zwischen 35000 und 32000 a B.P. Die M II-Moränen befinden sich immer über dem fBo1-Boden; ihr Alter konnte auf ca. 12000 a B.P. festgesetzt werden. Die M III-Moränen, die in drei Staffeln untergliedert werden können (HeINE 1976a), wurden zwischen 10000 und 9000 a B.P. gebildet. Sie überlagern im Bereich der Sierra Nevada de México einen Bims mit Andesit, der im Bereich des Popocatépetl (südliche Sierra Nevada) als markanter Leithorizont hervortritt und der sich bis zur NW-Flanke der Iztaccíhuatl verfolgen läßt. Es konnte nicht mit Sicherheit nachgewiesen werden, ob die Bimslinsen im Profil 1 (Abb. 4) dem Bims mit Andesit (P3 nach Mrentich 1974) des Popocatépetl entsprechen; die Untersuchungen von MieHLICH (1974) allerdings sprechen für die Korrelierung der Bimslinsen mit dem P3-Bims-Leithorizont des Popocatépetl, wodurch sich die Verknüpfung der Solifluktionshorizonte mit der Moränen-Stratigraphie erhärten würde. 
Die Solifluktionshorizonte bezeugen „periglaziale“ Prozesse zu verschiedenen Zeiten in Höhen um und unter $3000 \mathrm{~m}$. Anzeichen für einen Dauerfrostboden während der verschiedenen Solifluktionsphasen konnten in den Río Frio-Profilen nicht beobachtet werden, obgleich aus der Sierra Nevada fossile Eiskeile beschrieben werden (LoRENzo 1969; HeINE 1975). Vermutlich sind die Solifluktionshorizonte nicht an Dauerfrostboden gebunden gewesen, sondern entstanden, als während feuchter Phasen der - in einer schluffreichen $\mathrm{Ma}$ trix (= „toba“-Sedimente) eingebettete - Lavaschutt schwerkraftbedingt hangabwärts wanderte. Daher muß auch die Bildung der Solifluktionshorizonte zeitlich nicht unbedingt auf die relativ kurzen Phasen der starken Gletschervorstöße beschränkt gewesen sein, sondern die Solifluktionsdecken konnten sich eventuell auch schon vor und/oder nach den Gletschervorstößen bewegt haben, sofern dies die klimatischen Bedingungen zuließen.

\section{Schriftenverzeichnis}

Bloomfield, K. \& Valastro, S. (1974): Late Pleistocene Eruptive History of Nevado de Toluca Volcano, Central Mexico. - Bull. Geol. Soc. Amer., 85: 901-906, 3 Abb., 1 Tab.; Washington.

Bowler, J. M. (1975): Deglacial Events in Southern Australia: Their Age, Nature and Palaeoclimatic Significance. - Quaternary Studies - R. P. Suggate \& M. M. Cresswell (Eds.), Roy. Soc. New Zealand Bull., 13: 75-82, 3 Abb., 1 Tab.; Wellington.

Connwall, I. W. (1968): Outline of a stratigraphical "bridge" between the Mexico and Puebla basins. - Bull. Inst. Archaeol. Univ. London, 7: 89-140, 18 Abb.; London.

- (1970): Outline of a stratigraphical "bridge" between the Mexico and Puebla basins (Part two). - Bull. Inst. Archaeol. Univ. London, 8/9: 1-54, 6 Abb., 4 Tab.; London.

Heine, K. (1971): Fechas C14 de los sedimentos del volcán La Malinche. - Anu. Geogr., XI: 177-183, 2 Abb., 1 Tab.; Mexico.

- (1973a): Die jungpleistozänen und holozänen Gletschervorstöße am Malinche-Vulkan, Mexiko. - Eiszeitalter u. Gegenwart, 23/24: 46-62, 9 Abb., 1 Tab.; Ohringen.

- (1973b): Zur Glazialmorphologie und präkeramischen Archäologie des mexikanischen Hochlandes während des Spätglazials (Wisconsin) und Holozäns. - Erdkde., XXVII: 162-180, 5 Abb., 3 Phot., 3 Tab.; Bonn.

- (1974a): Nuevas observaciones de la estratigrafía Cuaternaria de la Región del Valle "PueblaTlaxcala“. - Comunicaciones (Proyecto Puebla Tlaxcala), 11: 1-3, 1 Abb.; Puebla, Pue. (Mex.).

- (1974b): Bemerkungen zu neueren chronostratigraphischen Daten zum Verhältnis glazialer und pluvialer Klimabedingungen. - Erdkde., 28: 303-312, 2 Abb., 1 Tab.; Bonn.

- (1975): Studien zur jungquartären Glazialmorphologie mexikanischer Vulikane - mit einem Ausblick auf die Klimaentwicklung. - Das Mexiko-Projekt der DFG, VII, 178 S., 39 Abb. 39 Bild., 9 Tab.; Wiesbaden (Steiner).

- (1976a): Schneegrenzdepressionen, Klimaentwicklung, Bodenerosion und Mensch im zentralmexikanischen Hochland im jüngeren Pleistozän und Holozän. - Z. Geomorph. N. F., Suppl.Bd. 24: 160-176, 5 Fig., 3 Phot., 1 Tab.; Berlin - Stuttgart.

- (1976b): Blockgletscher- und Blockzungen-Generationen am Nevado de Toluca, Mexiko. Die Erde, 1976: 330-352, 6 Fig., 4 Bilder, 1 Tab.; Berlin.

- (1977): Beobachtungen und Uberlegungen zur eiszeitlichen Depression von Schneegrenze und Strukturbodengrenze in den Tropen und Subtropen. - Erdkde., XXXI: 161-178, 8 Abb., 6 Phot., 1 Schema; Bonn.

- \& Heide-Weise, H. (1972): Estratigrafía del Pleistoceno Reciente y del Holoceno en el Volcán de la Malinche y Región Circunvecina. - Comunicaciones (Proyecto Puebla Tlaxcala), 5: 3-8, 2 Abb.; Puebla, Pue. (Mex.).

- \& - (1973): Jungquartäre Förderfolgen des Malinche-Vulkans und des Popocatépetl (Sierra Nevada, Mexiko) und ihre Bedeutung für die Glazialgeologie, Paläoklimatologie und Archäologie. - Münster. Forsch. Geol. Paläont., 31/32: 303-322, 2 Abb., 2 Tab.; Münster.

- \& Ohngemach, D. (1976): Die Pleistozän/Holozän-Grenze in Mexiko. - Münster. Forsch. Geol. Paläont., 38/39: 229-251, 7 Abb.; Münster.

- \& Schönhals, E. (1973): Entstehung und Alter der „toba“-Sedimente in Mexiko. — Eiszeitalter u. Gegenwart, 23/24: 201-215, 7 Abb.; O'hringen. 
KLAUS, D. (1973): Die eiszeitlichen und nacheiszeitlichen Klimaschwankungen im zentralmexikanischen Hochland und ihre Ursachen. - Erdkde., XXVII: 180-192, 5 Abb., 4 Tab.; Bonn.

Lorenzo, J. L. (1969): Condiciones periglaciares de las altas montañas de México. - Paleoecología (Dep. Prehist. INAH), 4: 45 S., 27 Fig., 5 Diagr.; Mexico.

MALDE, H. E. (o. J.): La Malinche Volcanic Ash Stratigraphy. - Manuskript.

Mienlich, G. (1974): Stratigraphie der jüngeren Pyroklastika der Sierra Nevada de México durch schwermineralanalytische und pedologische Untersuchungen. - Eiszeitalter u. Gegenwart, 25: 107-125, 6 Abb., 5 Tab.; Ohringen.

Mörner, N.-A. (1971): The Position of the Ocean Level during the Interstadial at about 30000 B.P. - A Discussion from a Climatic-Glaciologic Point of View. - J. Earth Sci. Canad., 8: 132-143, 4 Abb.; Montreal.

- (1972): The cold/warm changes during the last Ice Age. - Contr. Geol. Stockholm, XXIV (4): 51-77, 11 Abb., 3 Tab., 4 Taf.; Stockholm.

- (1973): Sea level variations and climatic fluctuations. - Coll. Internat. Centre Nat. Rech. Sci., 219: 135-141, 4 Abb.; Paris.

Schönhals, E. \& Werner, G. (1975): Acumulación ferrosa en los sedimentos volcánicos no compactos en Río Frio, Sierra Nevada. - Comunicaciones (Proyecto Puebla Tlaxcala), 12: 49-50, 2 Abb., 3 Phot.; Puebla - Mexico.

VAN Zinderen BAKKer, E. M. \& CoetzeE, J. A. (1972): A re-appraisal of late-Quaternary climate: evidence from tropical Africa. - Palaeoecol. of Africa, 7: 151-181, 21 Tab.; Kapstadt.

Manuskript eingeg. 14. 2. 1977. 